\title{
Front Matter: Volume 7937
}

, "Front Matter: Volume 7937," Proc. SPIE 7937, Ultrafast Phenomena in Semiconductors and Nanostructure Materials XV, 793701 (20 April 2011); doi: 10.1117/12.894874

SPIE. Event: SPIE OPTO, 2011, San Francisco, California, United States 


\title{
PROCEEDINGS OF SPIE
}

\section{Ultrafast Phenomena in Semiconductors and Nanostructure Materials XV}

\author{
Kong-Thon Tsen \\ Jin-Joo Song \\ Markus Betz \\ Abdulhakem Y. Elezzabi \\ Editors
}

23-26 January 2011

San Francisco, California, United States

Sponsored and Published by

SPIE 
The papers included in this volume were part of the technical conference cited on the cover and title page. Papers were selected and subject to review by the editors and conference program committee. Some conference presentations may not be available for publication. The papers published in these proceedings reflect the work and thoughts of the authors and are published herein as submitted. The publisher is not responsible for the validity of the information or for any outcomes resulting from reliance thereon.

Please use the following format to cite material from this book:

Author(s), "Title of Paper," in Ultrafast Phenomena in Semiconductors and Nanostructure Materials $X V$, edited by Kong-Thon Tsen, Jin-Joo Song, Markus Betz, Abdulhakem Y. Elezzabi, Proceedings of SPIE Vol. 7937 (SPIE, Bellingham, WA, 2011) Article CID Number.

ISSN 0277-786X

ISBN 9780819484741

Published by

SPIE

P.O. Box 10, Bellingham, Washington 98227-0010 USA

Telephone +1 3606763290 (Pacific Time) · Fax +1 3606471445

SPIE.org

Copyright (c) 2011, Society of Photo-Optical Instrumentation Engineers

Copying of material in this book for internal or personal use, or for the internal or personal use of specific clients, beyond the fair use provisions granted by the U.S. Copyright Law is authorized by SPIE subject to payment of copying fees. The Transactional Reporting Service base fee for this volume is $\$ 18.00$ per article (or portion thereof), which should be paid directly to the Copyright Clearance Center (CCC), 222 Rosewood Drive, Danvers, MA 01923. Payment may also be made electronically through CCC Online at copyright.com. Other copying for republication, resale, advertising or promotion, or any form of systematic or multiple reproduction of any material in this book is prohibited except with permission in writing from the publisher. The CCC fee code is 0277-786X/11/\$18.00.

Printed in the United States of America.

Publication of record for individual papers is online in the SPIE Digital Library.

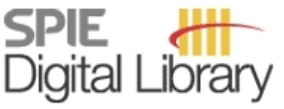

SPIEDigitalLibrary.org

Paper Numbering: Proceedings of SPIE follow an e-First publication model, with papers published first online and then in print and on CD-ROM. Papers are published as they are submitted and meet publication criteria. A unique, consistent, permanent citation identifier (CID) number is assigned to each article at the time of the first publication. Utilization of CIDs allows articles to be fully citable as soon as they are published online, and connects the same identifier to all online, print, and electronic versions of the publication. SPIE uses a six-digit CID article numbering system in which:

- The first four digits correspond to the SPIE volume number.

- The last two digits indicate publication order within the volume using a Base 36 numbering system employing both numerals and letters. These two-number sets start with 00, 01, 02, 03, 04 , 05, 06, 07, 08, 09, OA, OB ... 0Z, followed by 10-1Z, 20-2Z, etc.

The CID number appears on each page of the manuscript. The complete citation is used on the first page, and an abbreviated version on subsequent pages. Numbers in the index correspond to the last two digits of the six-digit CID number. 


\section{Contents}

ix Conference Committee

\section{SESSION 1 CARRIER DYNAMICS IN QUANTUM DOTS I}

793702 Ultrafast few-fermion dynamics in single self-assembled InGaAs/GaAs quantum dots (Invited Paper) [7937-01]

M. Betz, Technische Univ. Dortmund (Germany); M. Zecherle, C. Ruppert, E. C. Clark,

G. Abstreiter, J. J. Finley, Technische Univ. München (Germany)

793703 Ultrafast carrier capture and THz resonances in InGaAs quantum posts (Invited Paper) [7937-02]

D. Stehr, Univ. of California, Santa Barbara (United States) and Helmholtz-Zentrum Dresden-Rossendorf e.V. (Germany); C. M. Morris, Univ. of California, Santa Barbara (United States); D. Talbalyev, Los Alamos National Lab. (United States); M. Wagner, Helmholtz-Zentrum Dresden-Rossendorf e.V. (Germany); H. C. Kim, Univ. of California, Santa Barbara (United States); A. J. Taylor, Los Alamos National Lab. (United States); H. Schneider, Helmholtz-Zentrum Dresden-Rossendorf e.V. (Germany); P. M. Petroff, M. S. Sherwin, Univ. of California, Santa Barbara (United States)

793704 Ultrafast conditional carrier dynamics in semiconductor quantum dots (Invited Paper) [7937-03]

P. Borri, W. Langbein, Cardiff Univ. (United Kingdom)

\section{SESSION 2 CARRIER DYNAMICS IN QUANTUM DOTS II}

793707 Ultrafast density-and-temperature-dependent carrier dynamics in a quantum dots-in-a-well heterostructure (Invited Paper) [7937-06]

R. P. Prasankumar, Los Alamos National Lab. (United States); R. V. Shenoi, The Univ. of New Mexico (United States); J. Urayama, W. W. Chow, Sandia National Labs. (United States); S. Krishna, The Univ. of New Mexico (United States); A. J. Taylor, Los Alamos National Lab. (United States)

793708 Time resolved spectroscopy on quantum dots and graphene at the FELBE free-electron laser (Invited Paper) [7937-07]

S. Winnerl, D. Stehr, M. Wagner, H. Schneider, M. Helm, W. Seidel, P. Michel, Helmholtz-Zentrum Dresden Rossendorf e.V. (Germany); E. A. Zibik, B. A. Carpenter, N. E. Porter, M. S. Skolnick, L. R. Wilson, The Univ. of Sheffield (United Kingdom); T. Grange, R. Ferreira, G. Bastard, Lab. Pierre Aigrain, CNRS (France); M. Orlita, P. Plochocka, P. Kossacki, M. Potemski, Grenoble High Magnetic Field Lab. (France); M. Sprinkle, C. Berger, W. A. de Heer, Georgia Institute of Technology (United States)

793709 Enhancing slow- and fast-light effects in quantum dot semiconductor waveguides through ultrafast dynamics (Invited Paper) [7937-08]

Y. Chen, J. Mørk, Technical Univ. of Denmark (Denmark) 
7937 OB Ultrafast optical control of terahertz surface plasmons in subwavelength hole-arrays at room temperature (Invited Paper) [7937-10]

A. K. Azad, H.-T. Chen, A. J. Taylor, Los Alamos National Lab. (United States); W. Zhang, Oklahoma State Univ. (United States); J. F. O'Hara, Los Alamos National Lab. (United States)

7937 OD Plasmonics for THz frequency applications (Invited Paper) [7937-12]

E. Hendry, D. K. Polyushkin, T. H. Isaac, E. Stone, W. L. Barnes, The Univ. of Exeter (United Kingdom)

7937 OE THz patterned antennas for THz-TDS [7937-13]

P. Maraghechi, A. Y. Elezzabi, Univ. of Alberta (Canada)

\section{SESSION 4 LASER INDUCED STRUCTURAL MODIFICATIONS}

7937 OG Time-resolved probing of the acoustic field radiated by a single submicron gold particle (Invited Paper) [7937-15]

Y. Guillet, B. Audoin, CNRS, Univ. de Bordeaux (France); M. Ferrie, S. Ravaine, Ctr. de

Recherche Paul Pascal, CNRS (France)

\section{SESSION 5 DYNAMICS AT SURFACES}

$7937 \mathrm{OL} \quad 355 \mathrm{~nm}$ and $1064 \mathrm{~nm}$-pulse mixing to identify the laser-induced damage mechanisms in KDP (Invited Paper) [7937-20]

S. Reyné, G. Duchateau, Commissariat à l'Énergie Atomique (France); J.-Y. Natoli, Institut Fresnel, CNRS, Domaine Univ. St. Jérôme (France); L. Lamaignère, Commissariat à l'Énergie Atomique (France)

7937 OM Spin properties of interfaces with organic semiconductors studied by spin- and time-resolved two-photon photoemission (Invited Paper) [7937-21]

M. Cinchetti, K. Koffler, S. Steil, N. Großmann, M. Aeschlimann, Univ. of Kaiserslautern (Germany)

\section{SESSION 6 DYNAMICS IN LASER DEVICES}

7937 OP Ultrafast gain switching of THz quantum cascade lasers (Invited Paper) [7937-24] S. S. Dhillon, N. Jukam, D. Oustinov, R. Rungsawang, J. Madéo, Lab. Pierre Aigrain, CNRS Univ. Pierre et Marie Curie (France); S. Barbieri, C. Manquest, C. Sirtori, Univ. Paris 7-Denis Diderot (France); S. P. Khanna, E. H. Linfield, A. G. Davies, Univ. of Leeds (United Kingdom); J. Tignon, Lab. Pierre Aigrain, Ecole Normale Supérieure, CNRS (France)

$7937 \mathrm{OQ}$ Carrier dynamics investigation in quantum cascade lasers using Mid-IR ultrafast pulses [7937-25]

S. Liu, E. Lalanne, Univ. of Maryland, Baltimore County (United States); P. Q. Liu, Princeton Univ. (United States); X. Wang, AdTech Optics (United States); A. Johnson, Univ. of Maryland, Baltimore County (United States); C. Gmachl, Princeton Univ. (United States) 
7937 OS Passively mode-locked two section laser diode with intracavity dispersion control [7937-27] T. Schlauch, J. C. Balzer, M. R. Hofmann, Ruhr-Univ. Bochum (Germany); A. Klehr, G. Erbert, G. Tränkle, Ferdinand Braun Institute (Germany)

7937 OT Spin-controlled switching of lasing circular polarizations in (110)-oriented VCSELs [7937-28] N. Yokota, K. Ikeda, S. Koh, H. Kawaguchi, Nara Institute of Science and Technology (Japan)

7937 OU Injection currents in (110)-oriented GaAs/AIGaAs quantum wells: recent progress in theory and experiment (Invited Paper) [7937-29]

H. T. Duc, M. Pochwala, J. Förstner, T. Meier, Univ. Paderborn (Germany); S. Priyadarshi, A. M. Racu, K. Pierz, U. Siegner, M. Bieler, Physikalisch-Technische Bundesanstalt (Germany)

7937 OX One- and two-dimensional nonlinear THz spectroscopy on semiconductor nanostructures (Invited Paper) [7937-32]

M. Woerner, W. Kuehn, K. Reimann, T. Elsaesser, Max-Born-Institut für Nichtlineare Optik und Kurzzeitspektroskopie (Germany); R. Hey, Paul-Drude-Institut für Festkörperelektronik (Germany)

\section{CARRIER DYNAMICS IN NITRIDES}

7937 OY Carrier dynamics of $\mathbf{M g}$-doped indium nitride (Invited Paper) [7937-33]

H. Ahn, C.-C. Hong, National Chiao Tung Univ. (Taiwan); Y.-L. Hong, S. Gwo, National Tsing Hua Univ. (Taiwan)

793710 Ultrafast hot carrier dynamics in InN epitaxial films (Invited Paper) [7937-35] T.-C. Tsai, C.-F. Chang, C.-W. Kuo, C.-Y. Chang, National Taiwan Ocean Univ. (Taiwan); S. Gwo, National Tsing Hua Univ. (Taiwan)

793711 Long room-temperature electron spin lifetimes in bulk cubic GaN [7937-36] J. H. BuB, J. Rudolph, Ruhr-Univ. Bochum (Germany); T. Schupp, D. J. As, K. Lischka, Univ. of Paderborn (Germany); D. Hägele, Ruhr-Univ. Bochum (Germany)

793712 Photoexcited carrier relaxation dynamics of InN films and nanocolumns (Invited Paper) [7937-37]

M. Hashimoto, K. Fukunaga, Sophia Univ. (Japan); H. Kunugita, Sophia Univ. (Japan) and CREST (Japan); J. Kamimura, Sophia Univ. (Japan); A. Kikuchi, K. Kishino, K. Ema, Sophia Univ. (Japan) and CREST (Japan)

793713 Optical studies on $\mathbf{I n}_{\mathbf{x}} \mathbf{G a}_{1-\mathrm{x}} \mathbf{N}$ quantum disks (Invited Paper) [7937-38] M. J. Holmes, Univ. of Oxford (United Kingdom); Y. S. Park, Univ. of Oxford (United Kingdom) and Pohang Univ. of Science and Technology (Korea, Republic of); X. Wang, C. C. S. Chan, A. F. Jarjour, J. Luo, J. H. Warner, Univ. of Oxford (United Kingdom); H. A. R. El-Ella, R. A. Oliver, Univ. of Cambridge (United Kingdom); R. A. Taylor, Univ. of Oxford (United Kingdom) 
793715 Time-resolved x-ray scattering (Invited Paper) [7937-40]

D. Schick, Univ. Potsdam (Germany); C. von Korff Schmising, Lund Univ. (Sweden); A. Bojahr, M. Kiel, P. Gaal, M. Bargheer, Univ. Potsdam (Germany)

793716 Quasi-ballistic thermal transport from nanoscale interfaces observed using ultrafast coherent soft x-ray beams (Invited Paper) [7937-41]

M. E. Siemens, Univ. of Denver (United States) and Univ. of Colorado at Boulder (United States); Q. Li, R. Yang, Univ. of Colorado at Boulder (United States); K. A. Nelson, Massachusetts Institute of Technology (United States); E. H. Anderson, Lawrence Berkeley National Lab. (United States); M. M. Murnane, H. C. Kapteyn, Univ. of Colorado at Boulder (United States)

\section{SESSION 9 NANOPLASMONICS}

793719 Amplification of surface plasmons (Invited Paper) [7937-44]

I. De Leon, P. Berini, Univ. of Ottawa (Canada)

\section{SESSION 10 CARRIER DYNAMICS IN QUANTUM DOTS III}

7937 1C Robust quantum dot exciton preparation via adiabatic passage with frequency-swept laser pulses (Invited Paper) [7937-47]

B. Urbaszek, Lab. de Physique et Chimie des Nano-Objets, CNRS, Univ. de Toulouse (France); C.-M. Simon, Lab. de Physique et Chimie des Nano-Objets, CNRS, Univ. de Toulouse (France) and Lab. Collisions Agregats Reactivite, CNRS, Univ. Toulouse (France); T. Belhadj, Lab. de Physique et Chimie des Nano-Objets, CNRS, Univ. de Toulouse (France); B. Chatel, Lab. Collisions Agregats Reactivite, CNRS, Univ. Toulouse (France); T. Amand, P. Dalgarno, Lab. de Physique et Chimie des Nano-Objets, CNRS, Univ. de Toulouse (France); O. Krebs, Lab. de Photonique et Nanostructures (France); R. J. Warburton, Heriot-Watt Univ. (United Kingdom) and Univ. of Basel (Switzerland); A. Lemaitre, Lab. de Photonique et Nanostructures (France); X. Marie, P. Renucci, Lab. de Physique et Chimie des Nano-Objets, CNRS, Univ. de Toulouse (France)

7937 1D Photon statistics and phonon signatures in the quantum light emission from semiconductor quantum dots (Invited Paper) [7937-48]

A. Carmele, J. Kabuss, M. Richter, A. Knorr, Technische Univ. Berlin (Germany)

7937 IF Two-photon excited fluorescence from colloidal quantum dots on SiN photonic crystals (Invited Paper) [7937-50]

$X$. Xu, Institute of Semiconductors (China); T. Yamada, National Institute of Information and Communications Technology (Japan); S. Yokoyama, Kyushu Univ. (Japan)

7937 IG Förster resonance energy transfer in mixed-size CdTe quantum dots with optimized donor-acceptor concentration ratio (Invited Paper) [7937-51]

Y. C. Lin, W. C. Chou, National Chiao Tung Univ. (Taiwan); A. S. Susha, A. L. Rogach, City Univ. of Hong Kong (Hong Kong, China) 
$7937 \mathrm{lH} \quad$ Novel terahertz emission devices based on efficient optical frequency conversion in GaAs/AIAs coupled multilayer cavity structures on high-index substrates (Invited Paper) [7937-52]

T. Kitada, F. Tanaka, T. Takahashi, K. Morita, T. Isu, Univ. of Tokushima (Japan)

7937 1M Fiber-optic Cherenkov radiation excited by few-cycle pulses (Invited Paper) [7937-57]

G. Chang, L.-J. Chen, F. X. Kärtner, Massachusetts Institute of Technology (United States)

\section{SESSION 12 DYNAMICS IN STRONGLY CORRELATED MATERIALS}

793710 Photocarrier recombination dynamics of $\mathrm{SrTiO}_{3}$ (Invited Paper) [7937-59]

Y. Yamada, Y. Kanemitsu, Kyoto Univ. (Japan)

7937 IP Electron relaxation in metals and high-Tc superconductors on the 10-fs timescale (Invited Paper) [7937-60]

D. Brida, Politecnico di Milano (Italy); C. Gadermaier, Josef Stefan Institute (Slovenia);

D. Polli, Politecnico di Milano (Italy); V. V. Kabanov, D. Mihailovic, Josef Stefan Institute

(Slovenia); G. Cerullo, Politecnico di Milano (Italy)

\section{SESSION 13 CARRIER DYNAMICS IN CARBON NANOMATERIALS}

7937 IR Microscopic theory of ultrafast processes in carbon nanomaterials (Invited Paper) [7937-62] E. Malic, E. Bobkin, T. Winzer, C. Köhler, T. Watermann, M. Hirtschulz, A. Knorr, Technische Univ. Berlin (Germany)

7937 IU Ultrafast exciton and charge transfer in small aggregates of carbon nanotubes (Invited Paper) [7937-65]

L. Lüer, IMDEA Nanociencia (Spain) and Politecnico di Milano (Italy); J. Crochet, Los Alamos National Lab. (United States) and Univ. Würzburg (Germany); S. Hoseinkhani, Politecnico di Milano (Italy); T. Hertel, Univ. Würzburg (Germany); G. Cerullo, G. Lanzani, Politecnico di Milano (Italy)

\section{SESSION 14 ULTRAFAST ELECTRON DYNAMICS}

7937 IW Time-resolved nonlinear optical-holographic techniques for investigation of non-equilibrium carrier dynamics in semiconductors (Invited Paper) [7937-67] K. Jarašiūnas, Vilnius Univ. (Lithuania)

7937 IY Coherent oscillations between polariton vortex and anti-vortex states in an elliptical resonator [7937-72]

G. Nardin, Y. Léger, B. Pietka, F. Morier-Genoud, B. Deveaud-Plédran, Ecole Polytechnique Fédérale de Lausanne (Switzerland) 
$793721 \quad$ Pulse shaping properties of multi-layer volume holographic gratings [7937-71] A. Yan, L. Liu, J. Sun, Y. Zhou, Shanghai Institute of Optics and Fine Mechanics (China)

793722 Magnetic micro-trapping of excitons in multiple quantum wells system using local field minima [7937-73]

A. Abdelrahman, Edith Cowan Univ. (Australia); H. Kang, Gwangju Institute of Science and Technology (Korea, Republic of); M. Vasiliev, Edith Cowan Univ. (Australia); K. Alameh, Edith Cowan Univ. (Australia) and Gwangju Institute of Science and Technology (Korea, Republic of)

793723 Inhomogeneous spin-dependent spatial distribution of excitons in an integrated magnetic-multiple quantum wells system [7937-74]

A. Abdelrahman, Edith Cowan Univ. (Australia); H. Kang, Gwangju Institute of Science and Technology (Korea, Republic of); M. Vasiliev, Edith Cowan Univ. (Australia); K. Alameh, Edith Cowan Univ. (Australia) and Gwangju Institute of Science and Technology

(Korea, Republic of); Y. T. Lee, Gwangju Institute of Science and Technology

(Korea, Republic of)

Author Index 


\title{
Conference Committee
}

\author{
Symposium Chair
}

Liang-Chy Chien, Kent State University (United States)

Symposium Cochairs

E. Fred Schubert, Rensselaer Polytechnic Institute (United States)

Klaus P. Streubel, OSRAM GmbH (Germany)

Program Track Chair

James G. Grote, Air Force Research Laboratory (United States)

\section{Conference Chairs}

Kong-Thon Tsen, Arizona State University (United States)

Jin-Joo Song, University of California, San Diego (United States)

Markus Betz, Technische Universität Dortmund (Germany)

Abdulhakem Y. Elezzabi, University of Alberta (Canada)

\section{Program Committee}

Mischa Bonn, FOM Institute for Atomic and Molecular Physics

(Netherlands)

Yujie J. Ding, Lehigh University (United States)

Jan A. Gaj, University of Warsaw (Poland)

Rupert Huber, Universität Konstanz (Germany)

Robert A. Kaindl, Lawrence Berkeley National Laboratory

(United States)

Jacek Kasprzak, Cardiff University (United Kingdom)

Dai-Sik Kim, Seoul National University (Korea, Republic of)

Torsten Meier, Universität Paderborn (Germany)

Walter Pfeiffer, Universität Bielefeld (Germany)

Chi-Kuang Sun, National Taiwan University (Taiwan)

Fabrice Vallee, Université Claude Bernard Lyon 1 (France)

Hailin Wang, University of Oregon (United States)

Klaas Wynne, University of Strathclyde (United Kingdom)

Chih-Chung Yang, National Taiwan University (Taiwan) 
Carrier Dynamics in Quantum Dots I

Jin-Joo Song, University of California, San Diego (United States)

Carrier Dynamics in Quantum Dots II

Rupert Huber, Universität Konstanz (Germany)

Markus Betz, Technische Universität Dortmund (Germany)

Terahertz Plasmonics

Chih-Chung Yang, National Taiwan University (Taiwan)

Laser Induced Structural Modifications

Kong-Thon Tsen, Arizona State University (United States)

Dynamics at Surfaces

Yujie J. Ding, Lehigh University (United States)

Dynamics in Laser Devices

Robert A. Taylor, University of Oxford (United Kingdom)

Ultrafast Coherent Spectroscopy

Walter Pfeiffer, Universität Bielefeld (Germany)

Abdulhakem Y. Elezzabi, University of Alberta (Canada)

Carrier Dynamics in Nitrides

Torsten Meier, Universität Paderborn (Germany)

Ultrafast Electron/X-ray Diffraction

Markus Betz, Technische Universität Dortmund (Germany)

Nanoplasmonics

Jan A. Gaj, University of Warsaw (Poland)

Carrier Dynamics in Quantum Dots III

Chunlei Guo, University of Rochester (United States)

Nonlinear Optical Processes

Fabrice Vallee, Université Claude Bernard Lyon 1 (France)

Abdulhakem Y. Elezzabi, University of Alberta (Canada)

Dynamics in Strongly Correlated Materials

Guoqing Chang, Massachusetts Institute of Technology (United States)

Jin-Joo Song, University of California, San Diego (United States) 
Carrier Dynamics in Carbon Nanomaterials

Chi-Kuang Sun, National Taiwan University (Taiwan)

Ultrafast Electron Dynamics

Markus Betz, Technische Universität Dortmund (Germany) 
Downloaded From: https://www.spiedigitallibrary.org/conference-proceedings-of-spie on 26 Apr 2023

Terms of Use: https://www.spiedigitallibrary.org/terms-of-use 